\title{
Polydispersity and Heterogeneity of the Soluble Glycoprotein Isolated from Bovine Milk Fat Globule Membrane
}

\author{
Choemon Kanno, Makoto Shimizu and Kunio YamauchI \\ Department of Agricultural Chemistry, The University of Tokyo, \\ Bunkyo-ku, Tokyo 113, Japan \\ Received July 19, 1976
}

\begin{abstract}
Polydispersity and heterogeneity of the soluble glycoprotein isolated from bovine milk fat globule membrane were demonstrated by means of isoelectric focusing and immunoelectrophoresis.

The soluble glycoprotein showed a polydispersity on isoelectrofocusing gels, and its isoelectric $\mathrm{pH}$ ranged from 4.4 to 5.7. SDS-polyacrylamide gel electrophoresis of the polydispersed glycoprotein fractions separated by the isoelectric focusing suggested that the polydispersity was caused by various stages of intermolecular association of the constituent polypeptides.

Immunoelectrophoretic experiments showed that there were at least four different antigenic compounds. The appearance of the major precipitin lines suggested the polydispersity of the glycoprotein.

These results indicate that the soluble glycoprotein from milk fat globule membrane is not homogeneous although an apparent homogeneity was observed in some ordinary physicochemical analyses.
\end{abstract}

It is considered that milk fat globule membrane (MFGM) is a lipid-protein complex derived from plasma membrane of mammary secretory cell. ${ }^{1 \sim 3)}$ Many proteins of MFGM have been revealed on SDS-polyacrylamide gel

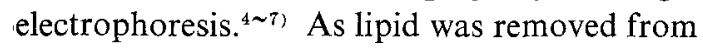
MFGM with chloroform-methanol mixture, most proteins became insoluble, while a small proportion of proteins was present in the soluble fraction. ${ }^{8)}$ The major protein in the soluble fraction was a glycoprotein and designated as the soluble glycoprotein. This glycoprotein had about $30 \%$ carbohydrate and showed apparent homogeneity on gel filtration, ultracentrifugal analysis and DEAE-Sephadex chromatography. ${ }^{8)}$ Furthermore, the soluble glycoprotein showed a single band on disc electrophoresis although the band was broad. ${ }^{8)}$ SDS-polyacrylamide gel electrophoretic study, however, indicated that the soluble glycoprotein consisted of at least eight polypeptides of approximate mol.wt. ranging from 10,000 to $88,000 .^{7)}$ It is, therefore, suggested that the eight constituent polypeptides form an apparently homogeneous glycoprotein complex.
In this report, we describe polydispersity and heterogeneity of the soluble glycoprotein complex revealed by means of isoelectrofocusing and immunoelectrophoresis.

\section{MATERIALS AND METHODS}

Preparation of the soluble glycoprotein. Soluble glycoprotein was prepared from bovine milk in accordance with the procedure described previously. ${ }^{8)}$ Specimens were purified by gel filteration on Bio-Gel A-5m (Bio-Rad Laboratories) for use in the experiments.

Isoelectric focusing. The gel medium was prepared to contain $6.0 \%$ acrylamide, $0.2 \%$ methylene bisacrylamide, $12.0 \%$ sucrose, $0.001 \%$ ribofravin, $0.1 \%$ $\mathrm{N}, \mathrm{N}, \mathrm{N}^{\prime}, \mathrm{N}^{\prime}$-tetramethylethylenediamine (TEMED) and $1.2 \%$ carrier ampholyte (Ampholine, LKB Instruments). As carrier ampholyte, $40 \%$ stock solutions of $\mathrm{pH} 3.5-10$ Ampholine and $\mathrm{pH} 2.5-4$ Ampholine were used to give $1.08 \%$ and $0.12 \%$, respectively. The protein sample $(1 \sim 2 \mathrm{mg}$ ) was dissolved directly in $10 \mathrm{ml}$ of the above mixture. The mixture was poured into glass columns $(5 \times 80 \mathrm{~mm})$ after exposure to vacuum suction for a few seconds to remove entrapped bubbles. The gel-protein mixture in glass columns was then polymerized under a fluorescent lamp for $2 \mathrm{hr}$ or more.

Isoelectric focusing was performed by the method of 
Finlayson and Chrambach. ${ }^{9)}$ The anode was placed in $0.2 \%(\mathrm{v} / \mathrm{v})$ sulfuric acid, and the cathode in $0.4 \%(\mathrm{v} / \mathrm{v})$ ethylenediamine solution. The current was regulated at $1 \mathrm{~mA}$ per tube until the potential reached $300 \mathrm{~V}$, which was then maintained for $8 \mathrm{hr}$. After electrofocusing, gels were washed, fixed by soaking in $12.5 \%$ trichloroacetic acid solution overnight and then stained with commassie blue $\mathrm{R}-250(\mathrm{CB})$ for protein and with periodic acid-Schiff (PAS) reagent for carbohydrate. Stained gels were scanned at $550 \mathrm{~nm}$ with a Gilford Spectrophotometer type 2400-2 having a linear transport system.

Determination of $\mathrm{pH}$ range was carried out by slicing unstained gel columns and by suspending the sliced sections in $1.0 \mathrm{ml}$ of water overnight. The $\mathrm{pH}$ was measured at room temperature with microelectrodes.

SDS-polyacrylamide gel electrophoresis. SDS-polyacrylamide gel electrophoresis was carried out by the method of Weber and Osborn ${ }^{10)}$ in $10 \%$ acrylamide gel concentration. Stained gels were scanned at $550 \mathrm{~nm}$ for coomassie blue and PAS with a Gilford Spectrophotometer type 2400-2.

Preparation of rabbit antiserum. Antiserum was produced in rabbits against the soluble glycoprotein of MFGM. The soluble glycoprotein in complete Freund adjuvant was injected into the foot pads or subcutaneously at two or three weeks intervals over a period of 6 months. The rabbits were bled on several occasions and the sera were pooled. Antiserum was concentrated by salting out at $2.0 \mathrm{M}$ ammonium sulfate ( $\mathrm{pH} 6.8$ ) and dialyzed against barbital buffer, $\mathrm{pH} 8.6, i=0.05$.

Immunodiffusion and immunoelectrophoresis. Double diffusion tests were performed by the procedure of Ouchterlony ${ }^{11)}$ with 1\% agar (Difco) Special AgarNoble, in barbital buffer, $\mathrm{pH} 8.6, i=0.1$.

Immunoelectrophoresis was carried out by the micromethod described by Scheidegger. ${ }^{12)}$ A $1.0 \mathrm{~mm}$ thick gel of $1.5 \%$ agar in barbital-acetate buffer, $\mathrm{pH} 8.6$, $i=0.05$, was moulded on a glass plate $(2.5 \mathrm{~cm} \times 7.0 \mathrm{~cm})$. Electrophoresis was performed at $6 \mathrm{~V}$ per $\mathrm{cm}$ for $90 \mathrm{~min}$. Diffusion was performed for $20 \mathrm{hr}$ at $25^{\circ} \mathrm{C}$.

The plates were then washed, and the precipitins were stained with Amino Black 10B.

\section{RESULTS}

Polydispersity of the soluble glycoprotein on isoelectrofocusing

A pattern of the soluble glycoprotein revealed on an isoelectrofocusing gel is shown in Fig. 1. Patterns stained with coomassie blue for protein and with PAS for carbohydrate were almost similar. By densitometric

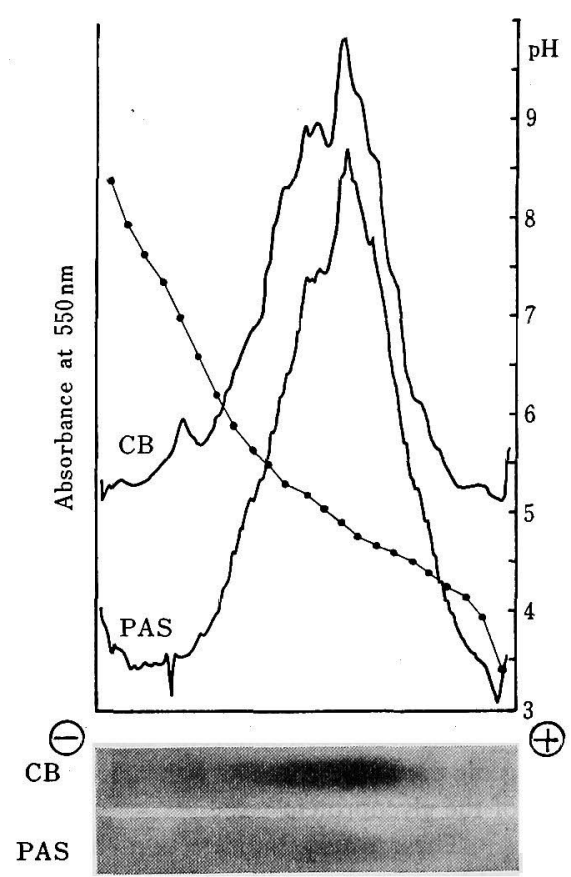

FIG. 1. Polydispersity of the Soluble Glycoprotein on Isoelectrofocusing.

Electrofocusing was performed at $300 \mathrm{~V}$ for $8 \mathrm{hr}$ at $4^{\circ} \mathrm{C}$. $1.2 \%$ of Ampholine (pH 3.5 10/pH 2.5 4; 9/1 (v/v)) was used. Protein and carbohydrate were stained with coomassie blue (CB) and periodic acid-Schiff (PAS), respectively.

scanning of the gels, a broad peak with some shoulders was observed, and isoelectric $\mathrm{pH}$ corresponding to this broad peak ranged from $\mathrm{pH} 4.4$ to 5.7. The apex of the peak was isoelectric $\mathrm{pH} 4.9$ in agreement with the isoelectric $\mathrm{pH}$ values, $\mathrm{pH} 3.6 \sim 5.0$, reported for the major boundary of the soluble fraction of MFGM observed upon free boundary electrophoresis. ${ }^{1)}$

The broad isoelectric $\mathrm{pH}$ range indicates the polydispersity of this glycoprotein. Such a polydispersed pattern suggests that the soluble glycoprotein is a mixture of glycoprotein molecules with slightly different isoelectric $\mathrm{pH}$, in spite of its apparent homogeneity in ultracentrifugal or chromatographic analysis. ${ }^{8)}$

\section{Constituent polypeptides of the polydispersed glycoprotein complex \\ The soluble glycoprotein is constructed of at}


least eight constituent polypeptides, which are detected on SDS-polyacrylamide gel electrophoresis as reported previously. ${ }^{71}$ We have considered that the polydispersity of the soluble glycoprotein molecule might mainly be caused by various stages of intermolecular association of these constituent polypeptides. To make clear this problem, the soluble

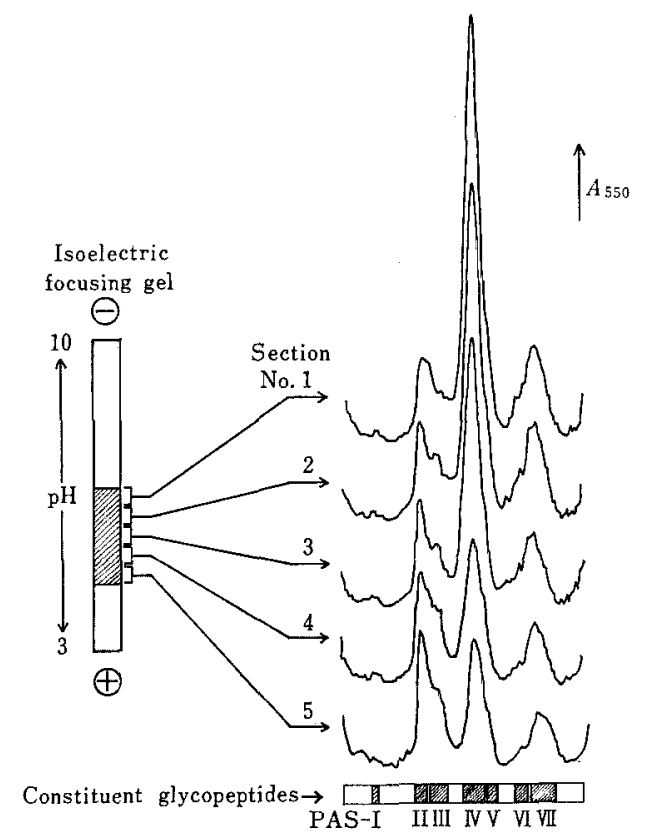

FIG. 2. SDS-polyacrylamide Gel Electrophoretic Patterns of the Soluble Glycoprotein Fractions Separated by Isoelectrofocusing.

Isoelectrofocusing gels were sliced into $3 \mathrm{~mm}$ sections. Five sections corresponding to the polydisersed glycoprotein band were extracted with water. The extracts were dialyzed against water, freeze-dried and subjected to SDS-polyacrylamide gel electrophoresis. Gels were stained with PAS and scanned at $550 \mathrm{~nm}$. glycoproteins of various isoelectric $\mathrm{pH}$ ranges were prepared by slicing the focused gel into five sections as indicated in Fig. 2, and extracting them with water. The extracted fractions were then subjected to SDS-polyacrylamide gel electrophoresis.

SDS-polyacrylamide gel electrophoretic patterns of the protein fractions extracted from the five sections are compared in Fig. 2. Ratios of the constituent glycopeptides, PASI $\sim$ VII, revealed by PAS staining were significantly different among the five fractions. Relative abundance of these constituent glycopeptides calculated from the scanning profiles is shown in Table I. The results indicate that the soluble glycoprotein with higher isoelectric pH contained a higher proportion of PAS$\mathrm{IV}+\mathrm{V}$ and a lower proportion of PAS-II + III, while one with lower isoelectric $\mathrm{pH}$ contained a higher proportion of PAS-II+III and a lower proportion of PAS-IV $+\mathrm{V}$. This tendency was not observed when coomassie blue staining was employed, since PAS-II, III, VI and VII were not detected with coomassie blue as found previously.7)

These results indicate that the constituent glycopeptides of the soluble glycoprotein exist in various stages of intermolecular association, which may cause the polydispersity on electrofocusing or disc electrophoresis. ${ }^{8)}$ Especially, the proportion of PAS-II+III to PAS-IV + - V is likely to affect the differences of isoelectric $\mathrm{pH}$ of the soluble glycoprotein complexes.

Heterogeneity of the soluble glycoprotein on immunodiffusion and immunoelectrophoresis The soluble glycoprotein showed three pre-

Table I. Relative Abundance of PAS-Components in the Fractions Extracted from the Polydispersed Band of the Soluble Glycoprotein

\begin{tabular}{lccccc}
\hline & \multicolumn{5}{c}{ Relative abundance $^{a)}$ (percentage of stain) } \\
\cline { 2 - 6 } Component $^{b)}$ & Section $^{c)} 1$ & 2 & 3 & 4 & 5 \\
\hline PAS-II + III & 17.3 & 21.0 & 25.4 & 38.1 & 43.0 \\
PAS-IV + V & 62.3 & 57.4 & 52.6 & 41.6 & 34.8 \\
PAS-VI +VII & 20.4 & 21.6 & 22.0 & 20.3 & 22.2 \\
\hline
\end{tabular}

a) Calculated from relative peak areas obtained by cutting and weighing sections of the staining profiles.

b) PAS-I was not determined.

c) Section $1 \sim 5$ correspond to those of Fig. 2 . 


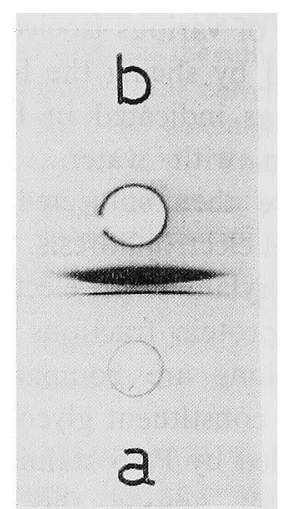

Fig. 3. Double Immunodiffusion of the Soluble Glycoprotein.

Diffusion was carried out for $90 \mathrm{hr}$ at $25^{\circ} \mathrm{C}$. Wells $\mathrm{a}$ and $\mathrm{b}$ contain antigen and antibody, respectively.

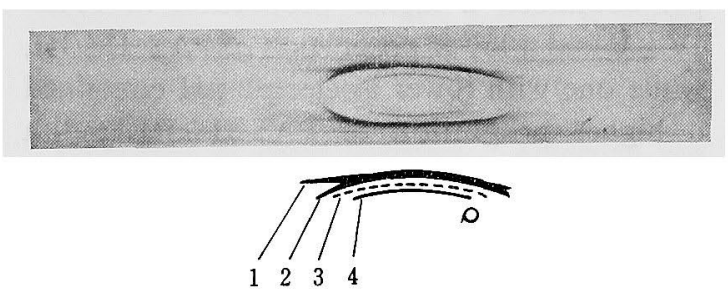

FIG. 4. Immunoelectrophoresis of the Soluble Glycoprotein.

Electrophoresis was carried out in barbital-acetate buffer, $\mathrm{pH} 8.6, i=0.05$, for $70 \mathrm{~min}$ at $6 \mathrm{~V} / \mathrm{cm}$. Diffusion was performed against antiserum of the soluble glycoprotein at room temperature for $20 \mathrm{hr}$.

cipitin lines on double-diffusion tests as shown in Fig. 3. On immunoelectrophoresis the major precipitin line on double-diffusion was further divided into at least two precipitin lines ( 1 and 2 ), which were particularly elongated and had diffused spurs (Fig. 4). These precipitin lines were stained with both coomassie blue and PAS. The appearance of the major precipitates suggests the presence of a series of glycoprotein substances of different mobilities but of the same antigenic specificity. This result may agree with the polydispersity of the soluble glycoprotein molecule shown by the isoelectrofocusing experiments. Two minor precipitates ( 3 and 4 ) were intensely stained with PAS, and this fact indicates that the antigens accounting for these precipitates were also glycoproteins.

\section{DISCUSSION}

It is well known that proteins constituting of biological membranes have a more or less hydrophobic area in their molecular structure which binds these proteins with hydrocarbon regions of membrane lipids. ${ }^{13,14)}$ Being delipidized, most of these proteins tend to form aggregates by hydrophobic interactions with each other and become insoluble. There are, however, examples of proteins which aggregate to form water-soluble complexes after delipidation. ${ }^{15,18)}$ Such complexes seem to be formed by mutual covering of the hydrophobic domains and to have a sufficiently polar surface to remain soluble.

Soluble glycoprotein obtained from MFGM by way of chloroform-methanol delipidation also seems to be one of such water-soluble complexes. This glycoprotein was apparently homogeneous and had a mol. wt. of $\left.139,000 .^{8}\right)$ However, when the molecular weights of the constituent polypeptides were summed, their total value was higher than $139,000 .^{7)}$ The present study of the combination of isoelectrofocusing with SDS-polyacrylamide gel electrophoresis indicated that the proportions of the constituent polypeptides in the soluble glycoprotein complex were not homogeneous, and this heterogeneity resulted in the considerable variation of the complex in its electric charge.

In addition, the presence of distinct molecules in the soluble glycoprotein fraction was observed on immunodiffusion and immunoelectrophoresis. There were at least four different precipitates, and two of them (3 and 4) were likely to be minor components in the soluble glycoprotein fraction. However, it is unknown how these molecules having distinct antigeneity participate in the formation of the soluble glycoprotein complex.

The soluble glycoprotein was shown to be a mixture of the glycoprotein complexes formed by various stages of intermolecular association among the constituent polypeptides. It is considered that these complexes are formed by mutual covering of the hydrophobic domains. However, the glycoprotein complex of more 
acidic isoelectric $\mathrm{pH}$ had a higher proportion of PAS-II+III and a lower proportion of PAS-IV $+V$. On the other hand, the glycoprotein complex having nearly neutral isoelectric $\mathrm{pH}$ had a higher proportion of PAS$\mathrm{IV}+\mathrm{V}$ and a lower proportion of PAS-II $+\mathrm{III}$ (Table I). This suggested that PAS-II and III were more acidic glycopeptides while PAS-IV and $\mathrm{V}$ were less acidic. Electrostatic interaction between these more acidic and less acidic glycopeptides might contribute to the formation of the soluble glycoprotein complex, in addition to the hydrophobic interaction between the hydrophobic domains of the polypeptides.

From these results it is supposed that the soluble glycoprotein is formed by random combination of constituent polypeptides, and contains a series of slightly different-sized molecules. The reported molecular weight of $\left.139,000^{8}\right)$ for the soluble glycoprotein, therefore, is the average molecular weight of the complexes.

Most polypeptides constituting the soluble glycoprotein complex are glycopeptides which are minor components in the total MFGM. ${ }^{8)}$ The hydrophobic areas of the glycopeptidesprobably smaller than those of polypeptides found in the insoluble fraction of delipidized MFGM - will be closed by the mutual covering, while their oligosaccharide chains will allow the solubility in the aqueous solution to remain.

It may be concluded that the soluble glycoprotein complexes prepared from MFGM by delipidation with organic solvent are not present originally in the native MFGM but are formed artificially by random association among polypeptides having somewhat hydrophobic domains and being rich in oligosaccharide chains.

\section{REFERENCES}

1) J. R. Brunner, "Fundamentals of Dairy Chemistry," 2nd ed., ed. by B. H. Webb, A. H. Johnson and J. A. Alford, the Avi Pub. Co., Inc., Westport, Connecticut, 1974, p. 474.

2) J. R. Brunner, "Structural and Functional Aspects of Lipoproteins in Living Systems,'” ed. by E. Tria and A. M. Scanu, Academic Press Inc., London and New York, 1969, p. 545.

3) S. Patton and T. W. Keenan, Biochim. Biophys. Acta, 415, 273 (1975).

4) D. Kobylka and K. L. Carraway, ibid., 288, 282 (1972).

5) M. Anderson, T. Cowston and G. C. Cheeseman, Biochem. J., 139, 653 (1974).

6) M. E. Mangino and J. R. Brunner, J. Dairy Sci., 58, 313 (1975).

7) M. Shimizu, C. Kanno and K. Yamauchi, Agric. Biol. Chem., 40, 1711 (1976).

8) C. Kanno, M. Shimizu and K. Yamauchi, ibid., 39, 1835 (1975).

9) G. R. Finlayson and A. Chrambach, Anal. Biochem., 40, 292 (1971).

10) K. Weber and M. Osborn, J. Biol. Chem., 244, 4406 (1969).

11) O. Ouchterlony, Acta Pathol. Microbiol. Scand., 25, 186 (1948).

12) J. J. Scheidegger, Intern. Arch. Allergy Appl. Immunol., 7, 103 (1955).

13) R. A. Capaldi and G. Vanderkooi, Proc. Nat. Acad. Sci. USA, 69, 930 (1972).

14) S. J. Singer and G. L. Nicolson, Science, 175, 720 (1972).

15) A. H. Maddy, Biochim. Biophys. Acta, 117, 193 (1966).

16) J. Azuma, M. Janado and K. Onodera, J. Biochem., 73, 1127 (1973). 